\title{
Recent Progress in Quantum Imaging and Ghost Imaging
}

\author{
Robert W. Boyd, Omar S. Magaña-Loaiza, Mehul Malik, Mohammad Mirhosseini, \\ Malcolm N. O'Sullivan, and Zhimin Shi
}

Institute of Optics, University of Rochester, Rochester, NY 14627 USA

We summarize recent work that demonstrates the utility of quantum effects for important technological applications in image science. We emphasize that what was considered to be "quantum weirdness" only a few years ago is today useful technology.

One area of recent advances is that of quantum imaging. Image formation making use of quantum states of light allows dramatic new possibilities in the field of image science. We review some of the conceptual possibilities afforded by quantum imaging and describe some recent work that displays some of these features. For example, we have shown that it is possible to impress an entire image onto a single photon and to distinguish among several objects even though they are illuminated by only a single photon [1]. We have also demonstrated that security protocols related to that of the BB84 protocol of QKD can be used to perform secure surveillance, that is, to ensure that image information in a scene of view has not been compromised [2]. Similar methods can be used for phase measurements in the field of quantum metrology. In one instance, we were able to establish a 16-fold increase in phase sensitivity by combining quantum and classical protocols [3].

As another example, we are currently developing a system to perform QKD at a high transmission rates by exploiting the transverse degree of freedom of the photon and specifically by encoding information in the Laguerre-Gauss modes. Specifically, we aim to transmit more than one classical bit of information per photon by making use of this large information capacity. This work entails studies aimed at determining the orbital angular momentum (OAM) spectrum of the light we generate [4], the development of sorters that can determine the value of the OAM of a single photon [5], and careful studies of the influence of atmospheric turbulence on the fidelity of a free-space QKD system [6].

\section{REFERENCES}

1. C. J. Broadbent, P. Zerom, H. Shin, J. C. Howell, and R. W. Boyd Phys. Rev. A 79033802 (2009).

2. M. Malik, O.S. Magaña-Loaiza, and R.W. Boyd, Appl. Phys. Lett. 101, 241103 (2012).

3. H. Shin, Omar S. Magaña-Loaiza, M. Malik, M.N. O’Sullivan, and R.W. Boyd, Optics Letters 21, 2816 (2013). 
4. M. Malik, S. Murugkar, J. Leach, and R.W. Boyd, Phys. Rev. A 86, 063806 (2012).

5. M. N. O’Sullivan, M. Mirhosseini, M. Malik, and R.W. Boyd, Optics Express 22, 24444 (2012).

6. B. Rodenburg, M. P. J. Lavery, M. Malik, M. N. O’Sullivan, M. Mirhosseini, D. J. Robertson, M. J. Padgett, and R. W. Boyd, Optics Letters 173735 (2012) 